Article

\title{
Synthesis of a Temperature-Sensitive Matrine-Imprinted Polymer and Its Potential Application for the Selective Extraction of Matrine from Radix Sophorae Tonkinensis
}

\author{
Minjie Jiang ${ }^{1}$, Lisheng Wang ${ }^{1, *}$, Xu Liu ${ }^{1}$, Hua Yang ${ }^{1}$, Fan Ren ${ }^{1}$, Lizhen Gan ${ }^{2}$ and \\ Weizhe Jiang ${ }^{2, *}$
}

1 School of Chemistry and Chemical Engineering, Guangxi University, 100 Daxue Road, Nanning 530004, China; E-Mails: jmj247742311@gmail.com (M.J.); wendaoliuxu@163.com (X.L.); yanghua6316@sina.com (H.Y.); fancyren@163.com (F.R.)

2 School of Pharmaceutical, Guangxi Medical University, 22 Shuangyong Road, Nanning 530021, China; E-Mail: welldone.123@163.com

* Authors to whom correspondence should be addressed;

E-Mails: jiangminjietp@outlook.com (L.W.); jiangweizhe6812@163.com (W.J.);

Tel./Fax: +86-771-4528-697 (L.W.); +86-771-5355-324 (W.J.).

Academic Editor: Jurriaan Huskens

Received: 23 November 2014 / Accepted: 26 January 2015 / Published: 4 February 2015

\begin{abstract}
A temperature-sensitive matrine-imprinted polymer was prepared in chloroform by free-radical cross-linking copolymerization of methacrylic acid at $60{ }^{\circ} \mathrm{C}$ in the presence of ethylene glycol dimethacrylate as the cross-linker, $N$-isopropyl acrylamide as the temperature-responsive monomer and matrine as the template molecule. Binding experiments and Scatchard analyses revealed that two classes of binding sites were formed on molecular imprinted polymer (MIP) at $50{ }^{\circ} \mathrm{C}$. Additionally, the thermoresponsive MIP was tested for its application as a sorbent material for the selective separation of matrine from Chinese medicinal plant radix Sophorae tonkinensis. It was shown that the thermoresponsive MIP displayed different efficiency in clean-up and enrichments using the SPE protocol at different temperatures.
\end{abstract}

Keywords: molecularly imprinted polymer; $N$-isopropyl acrylamide; matrine; ultrasonic extraction 


\section{Introduction}

Molecularly imprinted polymers (MIPs), which have selective recognition site(s) for a target compound or its structurally related analog(s), have been used as chromatographic media, such as LC stationary phases or adsorbents for solid-phase extraction (SPE) and solid-phase microextraction [1-3]. Cross-linked $\mathrm{N}$-substituted polyacrylamides are among the most widely studied polymeric materials used for the molecular imprinting of biomolecules such as proteins and DNA [4,5]. These polymers continue to receive much attention in the field of controlled drug delivery [6,7] because they can undergo a temperature-controlled volume phase transition in aqueous solutions.

Radix Sophorae tonkinensis is a widely used traditional Chinese herbal drug that possesses strong biological activity. Matrine (MT, Figure 1) plays an important role in the treatment of aches, tussis, tumors and arrhythmia and serves as an antifebrile, diuretic and antidote [8,9]. Few studies have reported on the separation of MT via a molecular imprinting technique [10-13]. Therefore, it is important to develop efficient methods for the separation and determination of MT from Chinese herbs.

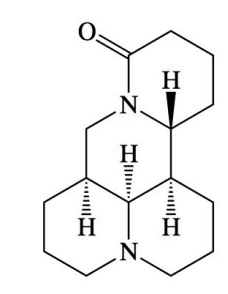

Matrine (MT)

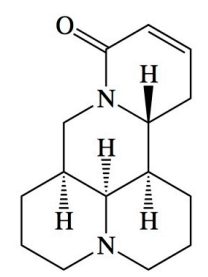

Sophocarpine (SC)

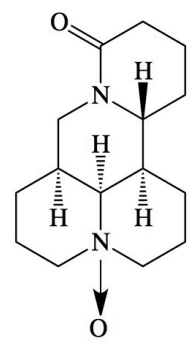

Oxymatrine (OMT)

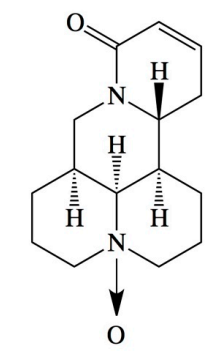

Oxysophocarpine (OSC)

Figure 1. Chemical structures of matrine (MT), oxymatrine (OMT), sophocarpine (SC) and oxysophocarpine (OSC).

So far, to the best of our knowledge, few have reported in literature the method in thermoresponsive MIP for selective extraction of MT with water as a solvent. The aims of this study were to prepare thermoresponsive imprinted polymers using ethylene glycol dimethacrylate (EDMA) as a cross-linking monomer and $N$-isopropyl acrylamide (NIPA) as a temperature-responsive monomer and to compare the polymers'recognition ability to that of various structurally rigid polymers. The temperature dependence of the recognition ability of the prepared thermoresponsive polymers and the application of the polymers as an adsorption phase coupled with the ultrasonic for the selective extraction of MT from radix Sophorae tonkinensis were also investigated. 


\section{Results and Discussion}

\subsection{Polymer Synthesis and Characterization}

The MT molecule has two hydrogen bonding sites, one on the amide group and another on the N atom of the heterocyclic group. The proper ratio of functional monomer to template molecules allows for a high adsorption capacity of the MIPs, whereas an excess of functional monomer enhances nonspecific adsorption. In the present work, we used a 1.5:4 ratio of template molecules to functional monomer as previously reported [11], and the binding of MT to thermosensitive imprinted polymers and structurally rigid polymers was compared. Normally, EDMA generates an imprinted polymer that is compact, inert and highly stable with respect to the rigidity of the polymer structure. Molecularly imprinted polymers and corresponding non-imprinted polymers, featuring NIPA and/or EDMA as a cross-linker, were created following a common protocol for MIP synthesis using the compositions listed in Table 1. The physical characteristics of the polymers were examined, and the results are summarized in Table 2. The results show that the pore diameter, pore volume and specific surface area of the NIPs and MIPs were not significantly different. When an excessive amount of NIPA was used during the pre-experiment stage, the polymers with soft texture and cannot be effectively reused were observed.

Table 1. Polymer composition. MIP: Molecularly imprinted polymer; NIP: Non-imprinted polymer.

\begin{tabular}{ccccccccc}
\hline Composition (mmol) & MIP1 & NIP1 & MIP2 & NIP2 & MIP3 & NIP3 & MIP4 & NIP4 \\
\hline Matrine (MT) & 1.5 & 0 & 1.5 & 0 & 1.5 & 0 & 1.5 & 0 \\
$\begin{array}{c}\text { Methacrylic acid (MAA) } \\
\text { Ethylene glycol dimethacrylate }\end{array}$ & 4 & 4 & 4 & 4 & 4 & 4 & 4 & 4 \\
$\begin{array}{c}\text { (EDMA) } \\
\text {-isopropyl acrylamide (NIPA) } \\
\mathbf{2 , 2} \text {-Azobis(2-isobutyronitrile) }\end{array}$ & 19.6 & 9.6 & 14.4 & 14.4 & 19.2 & 19.2 & 28.8 & 28.8 \\
(AIBN) & 1.9 & 1.9 & 1.9 & 1.9 & 1.9 & 1.9 & 1.9 & 1.9 \\
\hline
\end{tabular}

Table 2. Characteristics of the synthesized polymers.

\begin{tabular}{ccccc}
\hline Polymer & Particle Size $^{\text {a }}(\boldsymbol{\mu m})$ & Pore Diameter $(\mathbf{n m})$ & Pore Volume $(\boldsymbol{\mu L} / \mathbf{g})$ & BET Surface Area $\left(\mathbf{m}^{2} / \mathbf{g}\right)$ \\
\hline MIP1 & $5.52 \pm 0.03$ & $21.19 \pm 0.32$ & $2.73 \pm 0.18$ & $7.42 \pm 0.32$ \\
NIP1 & $6.01 \pm 0.04$ & $19.48 \pm 0.29$ & $1.91 \pm 0.11$ & $6.35 \pm 0.28$ \\
MIP2 & $5.62 \pm 0.03$ & $18.25 \pm 0.24$ & $1.53 \pm 0.09$ & $6.28 \pm 0.31$ \\
NIP2 & $6.33 \pm 0.05$ & $17.89 \pm 0.23$ & $1.65 \pm 0.10$ & $5.70 \pm 0.22$ \\
MIP3 & $7.72 \pm 0.06$ & $13.32 \pm 0.27$ & $1.27 \pm 0.08$ & $4.41 \pm 0.19$ \\
NIP3 & $9.37 \pm 0.08$ & $11.89 \pm 0.17$ & $1.19 \pm 0.09$ & $2.92 \pm 0.12$ \\
MIP4 & $8.23 \pm 0.08$ & $16.11 \pm 0.19$ & $1.02 \pm 0.07$ & $3.62 \pm 0.14$ \\
NIP4 & $12.78 \pm 0.14$ & $14.45 \pm 0.23$ & $0.76 \pm 0.04$ & $2.18 \pm 0.11$ \\
\hline
\end{tabular}

${ }^{\text {a }}$ Approximate mean particle size.

\subsection{Binding Experiments}

Parts a and $b$ of Figure 2 show the binding of MT to MIP (1, 2, 3 and 4) and NIP (1, 2, 3 and 4) at 25 and $50{ }^{\circ} \mathrm{C}$, respectively. At $25^{\circ} \mathrm{C}$, the amounts of MT bound to MIP gradually increased with the 
EDMA content and nothing different between MIPs and NIPs, but the amounts of MT bound to MIP were greater than the amounts of MT bound to NIP at $50{ }^{\circ} \mathrm{C}$. Temperature-sensitive polymers are known for their ability to reversibly swell and shrink in response to environmental temperature changes. Due to such properties, the reversible and reproducible adsorption of target molecules was realized for the MIPs. In this study, MIP2 demonstrated the best adsorption capacity. Figure 3 shows the binding isotherms of MT on MIP2 and NIP2 at $50{ }^{\circ} \mathrm{C}$; the binding amount increased gradually with an increase in MT concentration in the initial solution, but the amount of MT bound to MIP2 was greater than that bound to NIP2. Table 3 shows the $K_{\mathrm{d}}$ and $\mathrm{Q}_{\max }$ values determined by Scatchard analysis. These data reveal that two types of binding sites, high- and low-affinity sites, formed on MIP2 at $50{ }^{\circ} \mathrm{C}$, as previously reported [11], but at $25^{\circ} \mathrm{C}$, MIP2 and NIP2 exhibited similar properties.

Table 3. The binding characteristics of the thermoresponsive polymers at various temperatures. The correlation coefficients of the linear regression analyses were $0.955-0.999$.

\begin{tabular}{|c|c|c|c|c|c|c|c|c|}
\hline \multirow{3}{*}{$\begin{array}{c}\text { Temperature } \\
\left({ }^{\circ} \mathrm{C}\right)\end{array}$} & \multicolumn{4}{|c|}{ MIP2 } & \multicolumn{4}{|c|}{ NIP2 } \\
\hline & \multicolumn{2}{|c|}{ High-Affinity Site } & \multicolumn{2}{|c|}{ Low-Affinity Site } & \multicolumn{2}{|c|}{ High-Affinity Site } & \multicolumn{2}{|c|}{ Low-Affinity Site } \\
\hline & $\begin{array}{c}K_{\mathrm{d}} \pm \mathrm{SE}^{\mathrm{a}} \\
(\mu \mathrm{M})\end{array}$ & $\begin{array}{c}Q_{\max } \pm S E \\
(\mu \mathrm{mol} / \mathrm{g})\end{array}$ & $\begin{array}{c}K_{\mathrm{d}} \pm \mathrm{SE} \\
(\mu \mathrm{M})\end{array}$ & $\begin{array}{c}Q_{\max } \pm S E \\
(\mu \mathrm{mol} / \mathrm{g})\end{array}$ & $\begin{array}{c}K_{\mathrm{d}} \pm \mathrm{SE} \\
(\mu \mathrm{M}) \\
\end{array}$ & $\begin{array}{c}Q_{\max } \pm S E \\
(\mu \mathrm{mol} / g)\end{array}$ & $\begin{array}{c}K_{\mathrm{d}} \pm \mathrm{SE} \\
(\mu \mathrm{M})\end{array}$ & $\begin{array}{c}\mathrm{Q}_{\max } \pm \mathrm{SE} \\
(\mu \mathrm{mol} / \mathrm{g})\end{array}$ \\
\hline 50 & $507 \pm 63.5$ & $197 \pm 3.92$ & $4210 \pm 192$ & $422 \pm 66.3$ & - & - & $1720 \pm 277$ & $225 \pm 15.8$ \\
\hline 25 & - & - & $1620 \pm 227$ & $204 \pm 24.5$ & - & - & $1910 \pm 223$ & $202 \pm 16.4$ \\
\hline
\end{tabular}

a $\mathrm{SE}$ : standard error.
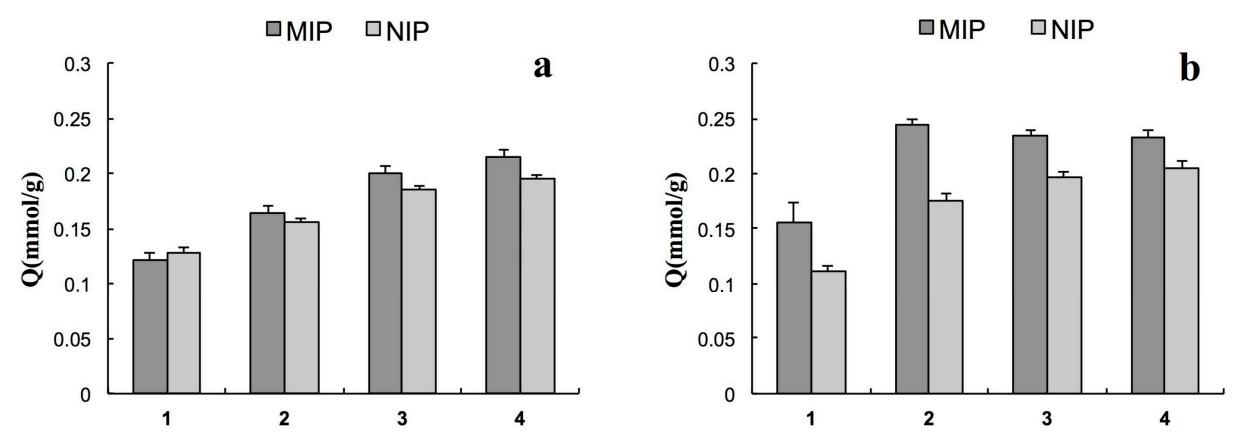

Figure 2. Binding of MT to molecular imprinted polymer (MIP) (1, 2, 3, and 4) and non-imprinted polymers (NIP) $(1,2,3$, and 4$)$ at $25^{\circ} \mathrm{C}$ (a) and $50{ }^{\circ} \mathrm{C}(\mathbf{b})($ mean $\pm \mathrm{SD}, n=3)$.

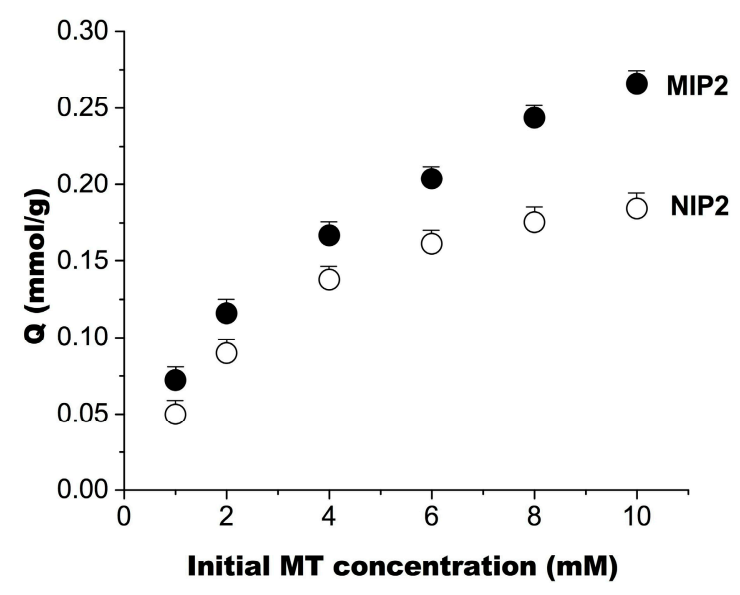

Figure 3. Binding isotherms of $\mathrm{MT}$ at $50{ }^{\circ} \mathrm{C}($ mean $\pm \mathrm{SD}, n=3)$. 


\subsection{Molecular Recognition Properties of MIP2}

Parts $\mathrm{a}$ and $\mathrm{b}$ of Figure 4 show the specific adsorption of MIP2 in a mixed solution of $10 \mathrm{mM} \mathrm{MT}$, OMT, SC and OSC at 50 or $25{ }^{\circ} \mathrm{C}$, respectively. MT and its structurally related compound, SC (13,14-dehydromatrine), were well recognized by MIP2, whereas neither OMT nor its structurally related compound, OSC (13,14-dehydrooxymatrine), were recognized at $50{ }^{\circ} \mathrm{C}$. Additionally, the adsorption amounts of SC, OMT and OSC increased slightly at $25^{\circ} \mathrm{C}$, most likely due to polymer swelling. These results indicate that MIP2 can selectively recognize MT at $50{ }^{\circ} \mathrm{C}$, but the recognition ability declines with decreasing temperature, which may be due to temperature-related swelling.
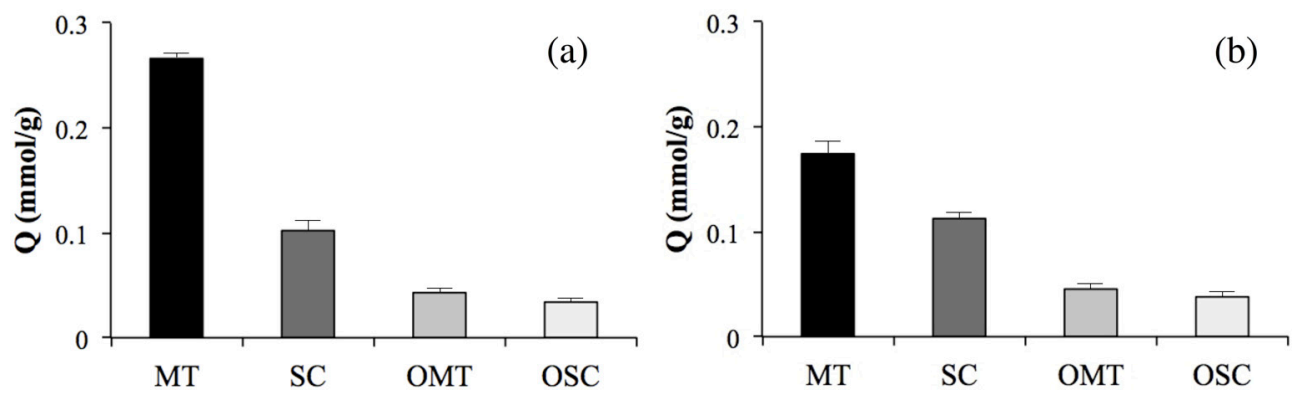

Figure 4. The specific adsorption of MIP2 at $50{ }^{\circ} \mathrm{C}(\mathbf{a})$ and $25^{\circ} \mathrm{C}(\mathbf{b})($ mean $\pm \mathrm{SD}, n=3)$.

\subsection{Temperature Effect on Recognition Ability}

The ability of the thermoresponsive polymer MIP2 to recognize a template molecule after a dynamic change in swelling was evaluated by equilibrium binding analysis. The experiment was performed at temperatures ranging from 25 to $70{ }^{\circ} \mathrm{C}$. Figure 5 shows how the adsorption pattern of MT varied with temperature for MIP2, with sorption increasing as a function of temperature. The temperature-dependent swelling of the thermoresponsive MIP2 is also shown in Figure 5. As the temperature increased from 35 to $40{ }^{\circ} \mathrm{C}$, it promoted binding of the template molecule to MIP2, whereas the corresponding NIP2 binding scarcely changed. The imprinting factor (the ratio of the amount of MT bound by the MIP to that bound by the corresponding NIP) of MIP2 was highest between 40 and $50{ }^{\circ} \mathrm{C}$; this range appeared to correspond to the transition temperature of the thermoresponsive MIP, which was higher than that of the MIP prepared by precipitation polymerization [11]. At temperatures beyond $50{ }^{\circ} \mathrm{C}$, it was observed that the increase in binding to the non-selective polymer was greater than the increase in binding to MIP, resulting in a decrease in the imprinting factor. The higher binding to the thermoresponsive MIP2 at high temperatures $\left(>50{ }^{\circ} \mathrm{C}\right)$ is likely the primary consequence of an increase in the non-specific adsorption of the template molecules.

\subsection{Recycling Times and Elution Process of MIP2}

Figure 6 shows the relationship between elution volume and the amount of MT. These results indicate that water at $25{ }^{\circ} \mathrm{C}$ easily eluted MT from MIP2, whereas it showed lower efficacy at $50{ }^{\circ} \mathrm{C}$. Thirty milliliters of water at $25^{\circ} \mathrm{C}$ completely eluted MT. These results indicate that MIP2 can maintain the configuration required for the specific adsorption of MT at $50{ }^{\circ} \mathrm{C}$. At $25{ }^{\circ} \mathrm{C}$, when the polymer is swelled with water, the adsorption mechanism changes from being specific to being nonspecific, thereby 
facilitating the elution of MT. The adsorption capacity was not significantly reduced after six cycles of repeated use.

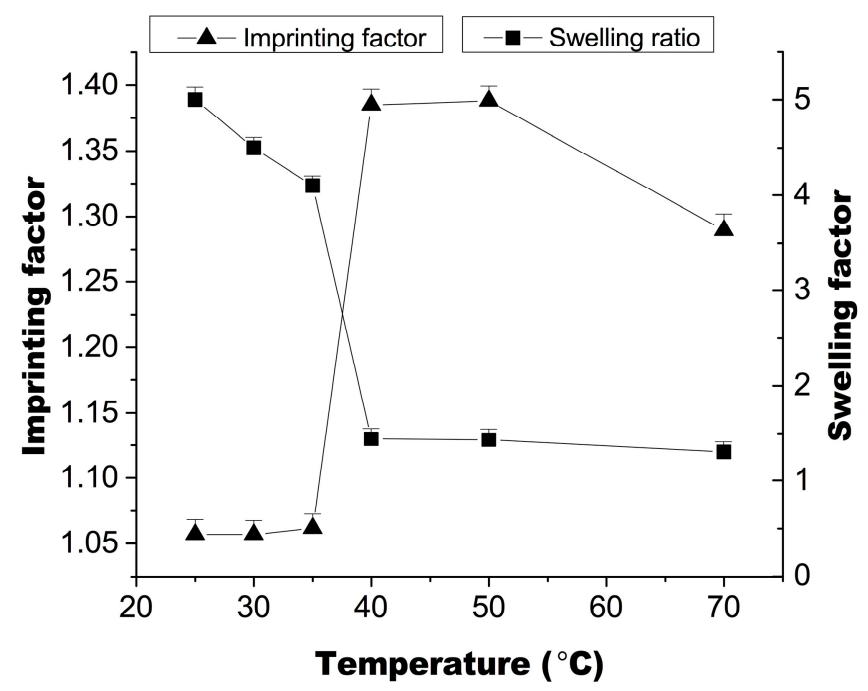

Figure 5. Binding affinity and swelling ratio of MIP2 (mean $\pm \mathrm{SD}, n=3)$.

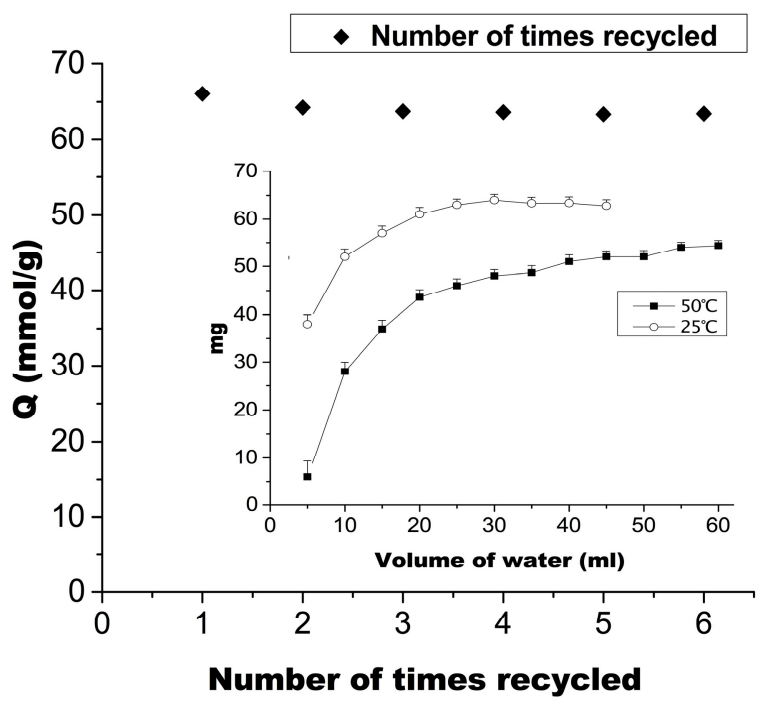

Figure 6. Number of times recycled and the relationship between elution volume and the quantity of MT extracted (mean $\pm \mathrm{SD}, n=3$ ).

\subsection{Selective Extraction of Matrine from Radix Sophorae Tonkinensis by MIP2 Coupled with} Ultrasonic Agitation and Desorption

Figure 7 shows the total amounts of MT extracted as a function of time. "Solution without MIP2", which represented the case in which MT was extracted from Radix Sophorae tonkinensis (before MIP2 extracted from radix Sophorae tonkinensis). On the contrary, "solution containing MIP2" which represented the case in which some MT was adsorbed onto MIP2 and some MT remained in solution (after MIP2 extracted from radix Sophorae tonkinensis, much higher than "solution containing NIP2"). These results suggest that MIP2 coupled with ultrasonic agitation can improve the dissolution rate of 
target compounds from Chinese medicinal herbs. Radix Sophorae tonkinensis contains approximately $2.43 \%$ matrine and oxymatrine (estimated by the Chinese Pharmacopoeia standard method).

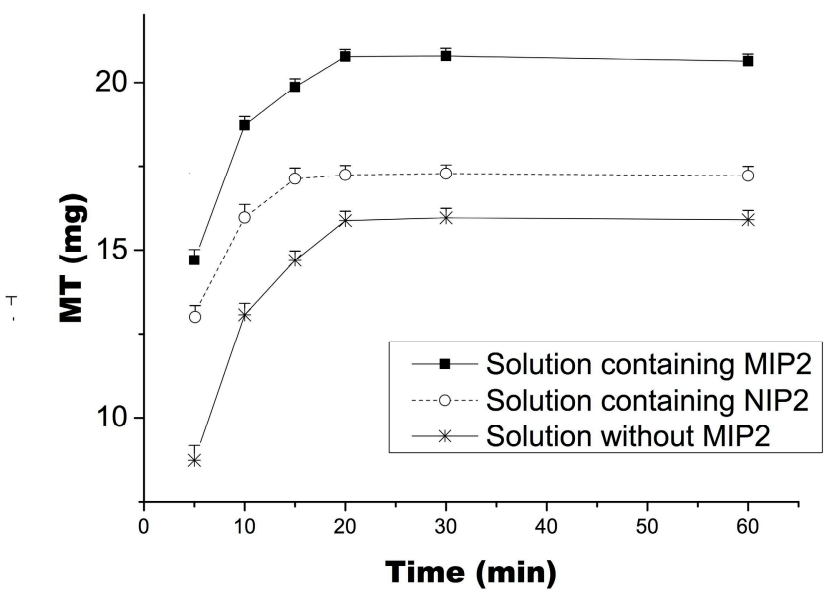

Figure 7. Total quantity of matrine (MT) extracted (mean $\pm \mathrm{SD}, n=3$ ).

In order to evaluate the ability of the MIP2 for obtaining of high purity of MT, three samples of adsorbed MIP2 sample (20, 30 or $60 \mathrm{~min})$ was eluted with $20,+10$ and $+10 \mathrm{~mL}$ of water $\left(50{ }^{\circ} \mathrm{C}\right)$ for removing impurities at first; for the selective adsorption of MT, the eluted MIP2 sample was then eluted with $30 \mathrm{~mL}$ of water $\left(25^{\circ} \mathrm{C}\right)$. Figure 8 shows chromatograms of the desorption of MIP2. As indicated, some compounds were retained on MIP2 when eluted with $20 \mathrm{~mL}$ of $50{ }^{\circ} \mathrm{C}$ water (A), whereas using more $10 \mathrm{~mL}$ of $50{ }^{\circ} \mathrm{C}$ water resulted in the removal of nearly all impurities (B). Further more elution with $10 \mathrm{~mL}$ of $50{ }^{\circ} \mathrm{C}$ water removed all impurities in addition to a small amount of MT (C). Figure 8D shows the chromatograms for the MIPs eluted with $30 \mathrm{~mL}$ of $25^{\circ} \mathrm{C}$ water from $\mathrm{C}$. The results indicate that, after the removal of impurities with hot water, the cold water elution of the target compounds appears to be feasible.

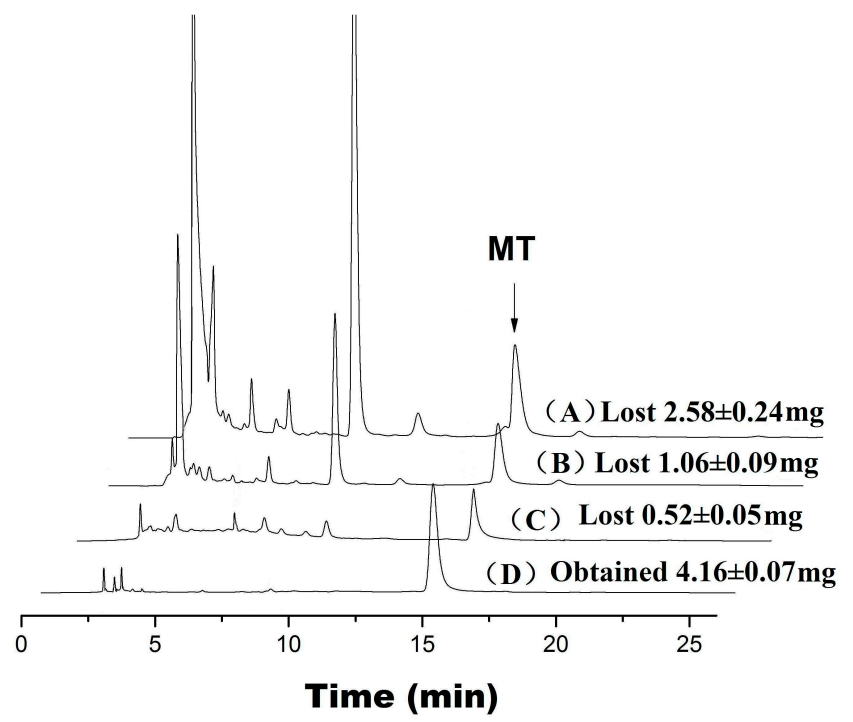

Figure 8. Desorption chromatograms of MIP2. A, B and C were eluted with $20,+10$ and $+10 \mathrm{~mL}$ of water with a temperature of $50{ }^{\circ} \mathrm{C}$ from saturated adsorption matrine samples; D were eluted with $30 \mathrm{~mL}$ of water with a temperature of $25^{\circ} \mathrm{C}$ from $\mathbf{C}$ (mean $\pm \mathrm{SD}, n=3$; Injection volume: $10 \mu \mathrm{L}$ ). 


\section{Experimental Section}

\subsection{Chemicals and Materials}

MT, OMT and OSC were purchased from the National Institute for the Control of Pharmaceutical and Biological Products (Beijing, China). SC was purchased from AvaChem Scientific (San Antonio, TX, USA). The chemical structures are shown in Figure 1. The Chinese herb radix Sophorae tonkinensis was purchased from a Chinese herbal drug store in Nanning, Guangxi Province, China. Methacrylic acid (MAA), $N$-isopropyl acrylamide (NIPA) and ethylene glycol dimethacrylate (EDMA) were purchased from Aldrich Chemical (Milwaukee, WI, USA). 2,2'-Azobis(2-isobutyronitrile) (AIBN) was supplied by the Special Chemical Reagent Factory of Nankai University (Tianjin, China). Methanol and chloroform were purchased from BDH Laboratory Supplies (Poole, UK). All solutions were prepared using high-purity water that had been purified by a Milli-Q plus system (Millipore, Molsheim, France).

\subsection{Instrumentation}

The liquid chromatography (LC) system used was composed of an LC-20AT pump, an SPD-20A UV-VIS Detector, an SIL-20A Autosampler, a CTO-20A Column Oven, and LC solution 20A (all from Shimadzu, Kyoto, Japan).

\subsection{Polymer Synthesis}

To obtain various structurally rigid polymers, four molecularly imprinted polymers (MIP1, MIP2, MIP3 and MIP4) and corresponding non-imprinted polymers (NIP1, NIP2, NIP3 and NIP4) were prepared using a thermal method involving free-radical polymerization according to previously reported methods [14]. To prepare these polymers, the polymerizing compositions listed in Table 1 were dissolved in $25 \mathrm{~mL}$ of chloroform. Subsequently, the polymeric mixtures were ultrasonically agitated under vacuum, purged with nitrogen for $5 \mathrm{~min}$ and polymerized by heating in a hot-air oven at $60{ }^{\circ} \mathrm{C}$ for $24 \mathrm{~h}$. The resulting polymers were crushed, ground and sieved through a 200-mesh sieve. The print molecules were eluted from the polymer particles by washing with three $500 \mathrm{~mL}$ portions of $10 \%(v / v)$ acetic acid in methanol and subsequently with three $500 \mathrm{~mL}$ portions of methanol.

\subsection{Characterization Methods}

The mean size and the size distribution of the prepared particles were determined at $25{ }^{\circ} \mathrm{C}$ using laser diffraction (Malvern Mastersizer, Worcester, UK) with water as the suspending medium. The mean of triplicate measurements for the same batch was determined. The degree of polymer swelling was determined as the ratio of the volume of the swollen polymer to that of the dry polymer in water using a calibrated measuring cylinder. The pore volume and specific surface area were measured via nitrogen adsorption/desorption techniques using a Coulter SA3100 series surface area and pore analyzer (Beckman Coulter, Inc., Brea, CA, USA), which enables pores sizes between 0.3 and $200 \mathrm{~nm}$ to be measured. The samples were degassed at $120{ }^{\circ} \mathrm{C}$, and a 50 -point pressure table was used. The surface area was determined from a Brunauer, Emmett and Teller (BET) plot, whereas the average 
pore diameter and the cumulative pore volume were obtained using a Barrett, Johner and Halenda (BJH) model of the adsorption isotherm.

\subsection{Binding Experiments}

The ability of the prepared MIPs to selectively recognize the template molecule relative to the prepared NIPs was evaluated in water after equilibration of the polymers with an MT solution. In a typical binding assay, the powdered polymer $(50 \mathrm{mg})$ of MIP or NIP was added to $5 \mathrm{~mL}$ of a water solution containing $8 \mathrm{mM}$ of MT or $5 \mathrm{~mL}$ of the pure solvent (blank). After shaking at 25 or $50{ }^{\circ} \mathrm{C}$ for $10 \mathrm{~h}$, an aliquot of each sample was taken and filtered through a $0.45 \mu \mathrm{m}$ membrane filter. The concentration of free MT in the supernatant was determined by LC with an Inertsil ODS-SP, $5 \mu \mathrm{m}$ column $\left(250 \mathrm{~mm} \times 4.6 \mathrm{~mm}\right.$ I.D.); a column temperature of $40{ }^{\circ} \mathrm{C}$; a mobile phase of $50 \mathrm{mM}$ phosphoric acid ( $\mathrm{pH}$ 2.3)-acetonitrile-methanol (940:50:10, $v / v / v)$ featuring $160 \mathrm{mM}$ sodium perchlorate; and a flow rate of $1.0 \mathrm{~mL} / \mathrm{min}$, with detection performed at $220 \mathrm{~nm}$. The quantity of drug in solution was determined by referencing a calibration curve. The amount of bound drug was obtained by subtracting the amount of free drug from the total amount of drug added.

The binding of MT to the thermoresponsive imprinted polymer MIP2 and corresponding non-imprinted polymer NIP2 was examined at six different temperatures $\left(25,30,35,40,45\right.$ and $\left.70{ }^{\circ} \mathrm{C}\right)$ with a binding assay protocol. The specific adsorption of MIP2 was examined in a mixed solution of $10 \mathrm{mM}$ of MT, OMT, SC and OSC at 25 or $50{ }^{\circ} \mathrm{C}$. The imprinting factor $(\alpha)$, which represents the effect of the imprinting process, was the ratio of the amount of substrate bound by the MIP to that bound by the corresponding NIP.

\subsection{Determination of the Thermoresponsive Polymers' Binding Characteristics}

The binding characteristics of a thermoresponsive imprinted polymer (MIP2) as well as the corresponding control polymer were examined at two different temperatures $\left(25\right.$ and $\left.50{ }^{\circ} \mathrm{C}\right)$ using $50 \mathrm{mg}$ samples of polymer in MT solutions with concentrations ranging from 1 to $10 \mathrm{mM}$. The amount of MT bound $(Q)$ was determined at each drug/polymer molar ratio $(R)$. The binding parameters were determined from the equation Bound/Free $=\left(B_{\max }-B\right) / K_{\mathrm{d}}$, where $K_{\mathrm{d}}$ is the equilibrium dissociation constant and $B_{\max }$ is the maximum number of binding sites, which were obtained from the slope and $\mathrm{x}$-intercept of the corresponding Scatchard plot, respectively. The association constant $\left(K_{\mathrm{a}}\right)$ was obtained as the reciprocal of $K_{\mathrm{d}}$. The mean MT binding constants were calculated based on results independently derived in triplicate.

\subsection{Recycling Times and Elution Process of MIP2}

Recycling time and elution experiments were performed using a glass SPE cartridge packed with $1 \mathrm{~g}$ MIP2 (MT adsorbed in $10 \mathrm{mmol} / \mathrm{L}$ MT solution for $10 \mathrm{~h}$ ). A water elution temperature of 25 or $50{ }^{\circ} \mathrm{C}$ was used, after the aqueous solution flowed through the SPE cartridge under unforced conditions. The respective effluents were collected in a conical flask. The mean value was obtained from triplicate measurements of the same batch. Then, the relationships between the elution volume and the quantity of MT were calculated. 


\subsection{Selective MT Extraction from Radix Sophorae Tonkinensis by MIP2 Coupled with Ultrasonic Agitation and Desorption}

Six samples were prepared via the following method. One gram of radix Sophorae tonkinensis was weighed and wrapped with 200-mesh nylon cloth and then extracted with $10 \mathrm{~mL}$ of water solution (containing 5\% sodium bisulfite, which enables the formation of SC, OSC and OMT in the form of MT, further increasing the yield) accompanied by $1 \mathrm{~g}$ of polymer (MIP2 or NIP2) in an ultrasonic bath at $50{ }^{\circ} \mathrm{C}$. A corresponding sample without polymer was also prepared. An aliquot of the extract from each of the six samples was passed through a $0.45 \mu \mathrm{m}$ membrane filter at 5, 10, 15, 20, 30 or $60 \mathrm{~min}$. Each saturated adsorption polymer (20,30 and 60 min of MIPs) was packed into a glass SPE cartridge and eluted with 20, 10 and $10 \mathrm{~mL}$ of water (at $50^{\circ} \mathrm{C}$ ) to remove impurities and then eluted with $30 \mathrm{~mL}$ of water to obtain the target compounds (at $25^{\circ} \mathrm{C}$ ). Other MIPs and NIPs, eluted with $60 \mathrm{~mL}$ of water (at $25^{\circ} \mathrm{C}$ ) to obtain the target compounds to calculate the dissolution rate of MT from Radix Sophorae tonkinensis.

\section{Conclusions}

The design and synthesis of thermally responsive materials for use in the extraction of MT were demonstrated in this study. An adsorption phase consisting of molecular recognition and thermally responsive elements was developed and evaluated for application using MIP2 as a sorbent material coupled with ultrasonic agitation for the extraction of MT using water as solvent from radix Sophorae tonkinensis. The thermoresponsive MIP displayed different efficiency in clean-up and enrichments using the SPE protocol at different temperatures. The results indicate that the cold water elution of the target compounds after the removal of impurities with hot water were feasible. This approach provides a new method for the extraction of MT in green process. In order to improve the imprinting factor, further functional monomers and polymerization method are currently in progress in our laboratory.

\section{Acknowledgments}

This work was supported by the Natural Science Foundation of China (No. 21262005) and the Natural Science Foundation of Guangxi (No. 2012GXNSFAA053158).

\section{Author Contributions}

Lisheng Wang and Weizhe Jiang conceived and designed experiments; Minjie Jiang, Xu Liu, Hua Yang, Fan Ren, and Lizhen Gan performed all experiments. Minjie Jiang and Lizhen Gan analyzed the data. Minjie Jiang wrote the paper. All authors read and approved the final manuscript.

\section{Conflicts of Interest}

The authors declare no conflict of interest.

\section{References}

1. Haginaka, J. Monodispersed, molecularly imprinted polymers as affinity-based chromatography media. J. Chromatogr. B Anal. Technol. Biomed. Life Sci. 2008, 866, 3-13. 
2. Turiel, E.; Martin-Esteban, A. Molecularly imprinted polymers for sample preparation: A review. Anal. Chim. Acta 2010, 668, 87-99.

3. Chen, L.; Xu, S.; Li, J. Recent advances in molecular imprinting technology: Current status, challenges and highlighted applications. Chem. Soc. Rev. 2011, 40, 2922-2942.

4. Rachkov, A.; Minoura, N. Towards molecularly imprinted polymers selective to peptides and proteins. The epitope approach. Biochim. Biophys. Acta Protein Struct. Mol. Enzymol. 2001, 1544, $255-266$.

5. Slinchenko, O.; Rachkov, A.; Miyachi, H.; Ogiso, M.; Minoura, N. Imprinted polymer layer for recognizing double-stranded DNA. Biosens. Bioelectron. 2004, 20, 1091-1097.

6. Byrne, M.E.; Park, K.; Peppas, N.A. Molecular imprinting within hydrogels. Adv. Drug Deliv. Rev. 2002, 54, 149-161.

7. Shibayama, M.; Nagai, K. Shrinking kinetics of poly ( $N$-isopropylacrylamide) gels T-jumped across their volume phase transition temperatures. Macromolecules 1999, 32, 7461-7468.

8. Xin, H.B.; Liu, S.F. Effects of matrine on myocardial contraction and arrhythmia in isolated heart atria. Acta Pharmacol. Sin. 1987, 8, 501-505.

9. Hu, Z.L.; Zhang, J.P.; Yu, X.B.; Lin, W.; Qian, D.H.; Wan, M.B. Effect of matrine on lipopolysaccharides/D-galactosamine-induced hepatitis and tumor necrosis factor release from macrophages in vitro. Zhongguo Yao Li Xue Bao 1996, 17, 351-353.

10. Lai, J.-P.; He, X.-W.; Jiang, Y.; Chen, F. Preparative separation and determination of matrine from the Chinese medicinal plant Sophora flavescens Ait by molecularly imprinted solid-phase extraction. Anal. Bioanal. Chem. 2003, 375, 264-269.

11. Funaya, N.; Haginaka, J. Matrine- and oxymatrine-imprinted monodisperse polymers prepared by precipitation polymerization and their applications for the selective extraction of matrine-type alkaloids from Sophora flavescens Aiton. J. Chromatogr. A 2012, 1248, 18-23.

12. Fu, Q.; Fang, Q.; Feng, B.; Sun, S.; Du, W.; Amut, E.; Xiao, A.; Chang, C. Matrine-imprinted monolithic stationary phase for extraction and purification of matrine from Sophorae flavescentis Ait. J. Chromatogr. B. Anal. Technol. Biomed. Life Sci. 2011, 879, 894-900.

13. Guo, Z.; Zhang, L.; Song, C.; Zhang, X. Molecularly imprinted solid-phase extraction of matrine from radix Sophorae tonkinensis. Analyst 2011, 136, 3016-3022.

14. Vlatakis, G.; Andersson, L.I.; Müller, R.; Mosbach, K. Drug assay using antibody mimics made by molecular imprinting. Nature 1993, 361, 645-647.

(C) 2015 by the authors; licensee MDPI, Basel, Switzerland. This article is an open access article distributed under the terms and conditions of the Creative Commons Attribution license (http://creativecommons.org/licenses/by/4.0/). 